\title{
Development of a Quality Assurance Process for the SoLid Experiment
}

\section{The SoLid Collaboration}

Y. Abreu, ${ }^{a}$ Y. Amhis, ${ }^{i}$ G. Ban, ${ }^{d}$ W. Beaumont, ${ }^{a}$ S. Binet, ${ }^{o}$ M. Bongrand, ${ }^{i}$ D. Boursette, ${ }^{i}$ B. C. Castle, ${ }^{j}$ H. Chanal, ${ }^{o}$ K. Clark, ${ }^{b}$ B. Coupé, ${ }^{k}$ P. Crochet, ${ }^{o}$ D. Cussans, ${ }^{b}$ A. De Roeck, ${ }^{a, e}$ D. Durand, ${ }^{d}$ M. Fallot, ${ }^{h}$ L. Ghys, ${ }^{k}$ L. Giot, ${ }^{h}$ K. Graves, ${ }^{g}$ B. Guillon, ${ }^{d}$ D. Henaff, ${ }^{h}$ B. Hosseini, ${ }^{g}$ S. Ihantola, ${ }^{g}$ S. Jenzer, ${ }^{i}$ S. Kalcheva, ${ }^{k}$ L. N. Kalousis, ${ }^{c}$ M. Labare,${ }^{f}$ G. Lehaut, ${ }^{d}$ S. Manley, ${ }^{b}$ L. Manzanillas, ${ }^{i}$ J. Mermans, ${ }^{k}$ I. Michiels, ${ }^{f}$ S. Monteil,${ }^{o}$ C. Moortgat, ${ }^{f, k}$ D. Newbold, ${ }^{b, m}$ J. Park, ${ }^{l}$ V. Pestel, ${ }^{d}$ K. Petridis, ${ }^{b}$ I. Piñera, ${ }^{a}$ L. Popescu, ${ }^{k}$ D. Ryckbosch, ${ }^{f}$ N. Ryder, ${ }^{j}$ D. Saunders, ${ }^{g}$ M.-H. Schune, ${ }^{i}$ M. Settimo, ${ }^{h}$ L. Simard, ${ }^{i, n}$ A. Vacheret, ${ }^{g}$ G. Vandierendonck, ${ }^{f}$ S. Van Dyck, ${ }^{k}$ P. Van Mulders, ${ }^{c}$ N. van Remortel, ${ }^{a}$ S. Vercaemer, ${ }^{a, c}$ M. Verstraeten, ${ }^{a}$ B. Viaud, ${ }^{h}$ A. Weber, ${ }^{j, m}$ F. Yermia ${ }^{h}$

${ }^{a}$ Universiteit Antwerpen, Antwerpen, Belgium

${ }^{b}$ University of Bristol, Bristol, $U K$

${ }^{c}$ Vrije Universiteit Brussel, Brussel, Belgium

${ }^{d}$ Normandie Univ, ENSICAEN, UNICAEN, CNRS/IN2P3, LPC Caen, 14000 Caen, France

${ }^{e}$ CERN, 1211 Geneva 23, Switzerland

${ }^{f}$ Universiteit Gent, Gent, Belgium

${ }^{g}$ Imperial College London, Department of Physics, London, United Kingdom

${ }^{h}$ Université de Nantes, IMT Atlantique, CNRS, Subatech, France

${ }^{i}$ LAL, Univ Paris-Sud, CNRS/IN2P3, Université Paris-Saclay, Orsay, France

${ }^{j}$ University of Oxford, Oxford, UK

${ }^{k}$ SCK-CEN, Belgian Nuclear Research Centre, Mol, Belgium

${ }^{l}$ Center for Neutrino Physics, Virginia Tech, Blacksburg, Virginia, 24061, USA

${ }^{m}$ STFC, Rutherford Appleton Laboratory, Harwell Oxford, United Kingdom

${ }^{n}$ Institut Universitaire de France, F-75005 Paris, France

${ }^{o}$ Université Clermont Auvergne, CNRS/IN2P3, LPC, F-63000 Clermont-Ferrand, France.

E-mail: manzanillas@lal.in2p3.fr, pestel@lpccaen.in2p3.fr 
Abstract: The SoLid experiment has been designed to search for an oscillation pattern induced by a light sterile neutrino state, utilising the $\mathrm{BR} 2$ reactor of $\mathrm{SCK} \bullet \mathrm{CEN}$, in Belgium.

The detector leverages a new hybrid technology, utilising two distinct scintillators in a cubic array, creating a highly segmented detector volume. A combination of $5 \mathrm{~cm}$ cubic polyvinyltoluene cells, with ${ }^{6} \mathrm{LiF}: \mathrm{ZnS}(\mathrm{Ag})$ sheets on two faces of each cube, facilitate reconstruction of the neutrino signals. Whilst the high granularity provides a powerful toolset to discriminate backgrounds; by itself the segmentation also represents a challenge in terms of homogeneity and calibration, for a consistent detector response. The search for this light sterile neutrino implies a sensitivity to distortions of around $O(10) \%$ in the energy spectrum of reactor $\bar{v}_{e}$. Hence, a very good neutron detection efficiency, light yield and homogeneous detector response are critical for data validation. The minimal requirements for the SoLid physics program are a light yield and a neutron detection efficiency larger than $40 \mathrm{PA} / \mathrm{MeV} /$ cube and $50 \%$ respectively. In order to guarantee these minimal requirements, the collaboration developed a rigorous quality assurance process for all 12800 cubic cells of the detector. To carry out the quality assurance process, an automated calibration system called CALIPSO was designed and constructed. CALIPSO provides precise, automatic placement of radioactive sources in front of each cube of a given detector plane (16×16 cubes). A combination of ${ }^{22} \mathrm{Na},{ }^{252} \mathrm{Cf}$ and AmBe gamma and neutron sources were used by CALIPSO during the quality assurance process. Initially, the scanning identified defective components allowing for repair during initial construction of the SoLid detector. Secondly, a full analysis of the calibration data revealed initial estimations for the light yield of over $60 \mathrm{PA} / \mathrm{MeV}$ and neutron reconstruction efficiency of $68 \%$, validating the SoLid physics requirements.

Keywords: Neutrino detector, Sterile neutrino, Neutron detectors (cold, thermal, fast neutrons), Particle identification methods, Calorimeters 


\section{Contents}

1 Introduction 1

2 Preparation of the SoLid Planes 3

3 The CALIPSO Calibration System 4

3.1 CALIPSO Design 4

3.2 CALIPSO Readout System 4

3.3 CALIPSO Monte Carlo 4

4 Light Yield Measurement $\quad 7$

4.1 Determining MPPC Operating Voltage 8

4.2 Cube Light Yield and Signal Reconstruction 9

$\begin{array}{ll}4.3 \text { Compton Edge Analytical Fit } & 11\end{array}$

$\begin{array}{lll}4.4 & \text { Kolmogorov-Smirnov Test } & 12\end{array}$

$\begin{array}{lll}4.5 & \text { Construction Adjustments } & 14\end{array}$

$\begin{array}{lll}4.6 & \text { Light Yield Results } & 14\end{array}$

5 Neutron Detection Efficiency 16

$\begin{array}{ll}5.1 \text { Nuclear Signal Reconstruction } & 16\end{array}$

$\begin{array}{lll}\text { 5.1.1 Neutron Trigger } & 16\end{array}$

$\begin{array}{ll}\text { 5.1.2 Neutron Particle Identification } & 17\end{array}$

$\begin{array}{lll}5.2 & \text { Neutron Detection Efficiency Estimation } & 17\end{array}$

$\begin{array}{lll}5.3 \text { Construction Adjustments } & 19\end{array}$

6 Discussion and Conclusions 20

\section{Introduction}

Previous short baseline (anti)-neutrino oscillation experiments have evidenced deficits in the observed number of (anti)-neutrino events, with respect to theoretical expectations [1-3].

The most relevant discrepancy for SoLid is the Reactor Antineutrino Anomaly (RAA) [3], which was first determined from re-evaluation of the antineutrino flux and spectra of nuclear reactors [4, 5]; and then confirmed by the RENO, Double Chooz, and Daya Bay experiments [6]. A possible interpretation of these deficits is the existence of oscillation effects induced by a light sterile neutrino state [7]. In recent years many experiments have been proposed and constructed in order to test the light sterile neutrino hypothesis as the origin of the RAA [8-13], and have also revealed a significant distortion in the $\bar{v}_{e}$ energy spectrum around $5 \mathrm{MeV}$ [14-17]. First hints suggest that this distortion is correlated with the reactor power [16] and might be associated with the ${ }^{235} \mathrm{U}$ fuel [18]. 
Recent analyses of the RAA suggest a sterile neutrino with a $\Delta m_{41}^{2}$ around either 1.3 or 1.7 $\mathrm{eV}^{2}[7,19]$. The oscillation of $\bar{v}_{e}$ 's into this new sterile state would induce distortions in the $\bar{v}_{e}$ spectrum measured at very short baselines $(L<10 \mathrm{~m})$, which should not exceed the $10 \%$ level [19].

The SoLid collaboration have developed a novel detection technique, utilising a highly granular, hybrid detector. As in all oscillation based reactor neutrino experiments, electron antineutrinos are detected by SoLid through the Inverse Beta Decay (IBD) process:

$$
\bar{v}_{e}+p \rightarrow e^{+}+n
$$

Within the detector volume of SoLid, polyvinyltoluene (PVT) cubes of $5 \times 5 \times 5 \mathrm{~cm}^{3}$ are combined with ${ }^{6} \mathrm{LiF}: \mathrm{ZnS}(\mathrm{Ag})$ screens, on two faces of each cube as shown in figure 1, to form volumetric pixels, or "voxels".

The PVT serves not only as the target for the IBD reaction, but also as a calorimeter for the positron, which produces a "prompt" signal; and as a moderator for the neutron of the IBD reaction. After thermalisation, the neutron will be captured and detected in the second scintillator, the ${ }^{6} \mathrm{LiF}: \mathrm{ZnS}(\mathrm{Ag})$ screens, taking advantage of its high neutron capture cross section, initiating a "delayed" signal.

After neutron capture, the Li nuclei will break-up, according to the following reaction:

$$
{ }_{3}^{6} \mathrm{Li}+n \rightarrow{ }_{1}^{3} \mathrm{H}+\alpha
$$

which has a Q-value of $4.78 \mathrm{MeV}$, which will predominantly be deposited in the surrounding $\mathrm{ZnS}$ scintillator.

Since both scintillators have distinct light emission properties, a powerful Pulse Shape Discrimination (PSD) algorithm can be used during analyses of events. The PSD is used in conjunction with a time coincidence between the prompt and delayed signals, to reconstruct the IBD candidate events.

The $\bar{v}_{e}$ energy is determined through reconstruction of the positron energy using the scintillation from the PVT as the prompt, electronic signal (ES); the ${ }^{6} \mathrm{LiF}: \mathrm{ZnS}$ provides data regarding the nuclear signal (NS).

In order to guarantee optical isolation and to enhance light collection, the cubic voxels are individually wrapped in Tyvek. Voxels are arranged into planes of $16 \times 16$ cubes, with 10 planes being grouped into one SoLid module. The SoLid detector includes 5 modules, for a total target mass of $1600 \mathrm{~kg}$. Both prompt and delayed signals are read out by the same network of wavelength shifting fibres and Multi-Pixel Photon Counters (MPPCs) [20]. A detailed description of the detector concept and the performance of the constructed SM1 prototype can be found in [12,21].

The granularity inherent in the hybrid voxel technology allows for clear identification of the neutrino signals, significantly reducing backgrounds. Constructing such a segmented detector provides a significant challenge in terms of homogeneity, light yield and neutron detection efficiency, which are of paramount importance in reactor-based searches for light sterile neutrinos, where oscillation effects are expected to be small. In order to have the sensitivity to these small effects, the SoLid minimal requirements include a light yield larger than $40 \mathrm{PA} / \mathrm{MeV} /$ cube in order to guarantee an energy resolution better than $16 \%$, and a neutron detection efficiency larger than $50 \%$ to achieve an IBD detection efficiency of about $30 \%$. 
To validate the necessary performance of the SoLid detector and to enable prompt identification of defective components, a quality assurance (QA) process was developed during construction. To this end, an automated calibration system called CALIPSO was constructed. CALIPSO provided an initial calibration for all 50 planes of SoLid before they were assembled into modules for the BR2 reactor site. Initial estimations of the light yield and neutron detection efficiency for all 12800 voxels were obtained and validated using gamma and neutron radioactive sources.

Section 2 of this paper describes the frame construction procedure, and section 3 describes in detail the design and performance of CALIPSO. Sections 4 and 5 detail the methods used to assess the light yield and neutron detection efficiency. Finally section 6 presents a discussion and conclusions on the quality assurance process of the SoLid experiment.

\section{Preparation of the SoLid Planes}

The SoLid detector is composed of 12800 fundamental cubic voxel units. Each voxel comprises a PVT cube of $5 \times 5 \times 5 \mathrm{~cm}^{3}$ in conjunction with two layers of $\sim 250 \mu \mathrm{m}$ thick ${ }^{6} \mathrm{LiF}: \mathrm{ZnS}(\mathrm{Ag})$, as illustrated in figure 1 . This voxel configuration was defined after a dedicated study to improve the light collection of the planar sub-assemblies [22].
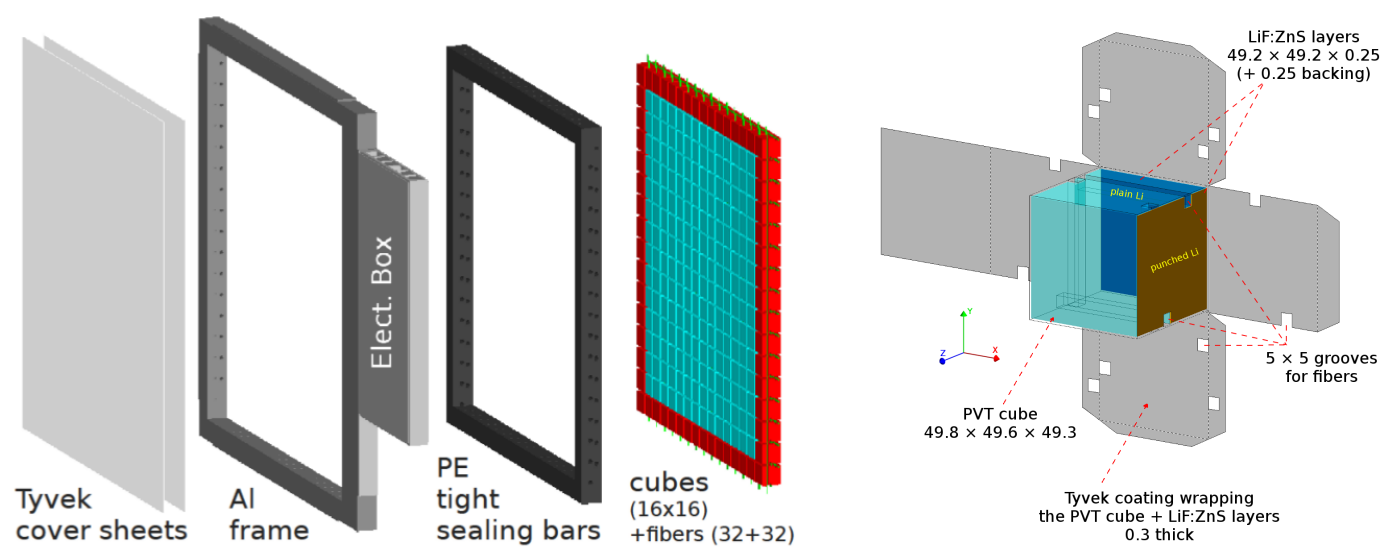

Figure 1. Left : Different components of a SoLid plane. Right : Sketch of a fundamental SoLid unit, a voxel.

During construction of the voxel units, each PVT cube is cleaned, measured and weighted. Metrics for the lithium sheets and Tyvek wrappings are added to the cube data to form a detailed assay of each voxel. The voxels are then tagged and stored in hermetic plastic boxes until mounting within their specific planes.

For each plane, an aluminium frame provides both mechanical support and a mounting point for the associated electronics. Within this frame a border of $5 \mathrm{~cm}$ thick polyethylene (PE) is used as both an internal neutron reflector and as external shielding. A Tyvek sheet is applied to one face of the plane, to offer lateral mechanical support to the voxels, and to provide additional optical isolation between planes. The voxels locations are recorded and linked to the assay database, before 64 optical fibres are threaded throughout the entire plane. This results in four fibres passing through each of the 256 voxels. Each fibre is coupled via 3D printed mountings; at one end to a mirror, 
and at the other to an MPPC, using optical grease for maximal light transmission. The supports are designed to adhere to the aluminium frames and are glued in place. Prior to cabling the 64 MPPCs to the readout electronics, a final sheet of Tyvek is layered over the open side to fully enclose the voxel array, as in figure 1.

Following assembly, each completed plane is calibrated in a light-tight room housing the CALIPSO robotic system.

\section{The CALIPSO Calibration System}

\subsection{CALIPSO Design}

The CALIPSO system was designed to perform a time efficient and accurate quality assurance process while constructing the SoLid detector.

The system is driven by a dedicated data acquisition system, which provides simultaneous control of the robots movement and data taking. CALIPSO has sub-millimetre precision $O(0.5)$ $\mathrm{mm}$ in the $\mathrm{XY}$ axes with adjustment in the $\mathrm{Z}$ axis being provided by a graduated rolling chassis, upon which the robotic armature is mounted. The CALIPSO system has been designed to operate in both neutron and gamma modes. In neutron mode the planes are placed between plates of polyethylene (brown plates in the figure 2) and a neutron source is placed inside a polyethylene collimator (see figure 3). In gamma mode, these PE plates are removed and a system for an external trigger is used (see figure 3).

\subsection{CALIPSO Readout System}

Acquisition of the MPPC signals was performed using a prototype electronics box placed on one side of the frames as shown in figure 2. The electronics box is composed of 2 parts: an analogue and a digital front-end. For the analogue front end, two boards serving 32 channels each, are used in each electronics box. These two boards provide each MPPC with the corresponding bias voltage, set according to a value controlled by the digital board. The analogue boards also amplify and shape the signals before transmitting to the digital board. The digital board includes the trigger, on a Field-Programmable Gate Array (FPGA) chip, and the Analogue-Digital Converter chips. The 8 Analogue-Digital Converters sample 8 channels each, at a rate of $40 \mathrm{MHz}$ with a 14-bit resolution $[23,24]$.

The CALIPSO calibration campaign facilitated testing of the prototype electronics box prior to manufacturing. Using data acquisition software designed for the fully assembled experiment, CALIPSO also served as a trigger and DAQ development platform.

\subsection{CALIPSO Monte Carlo}

A dedicated Monte-Carlo model (Geant4 based [25]), including CALIPSO and its direct environment was developed, in order to optimise the setup and the QA procedure. The ${ }^{22} \mathrm{Na}$ radioactive gamma source was simulated using the radioactive decay class from Geant4.

Accounting the ${ }^{22} \mathrm{Na}$ source activity, the exposure time per cube was set to 30 seconds, in order to guarantee at least $15000 \gamma$ interactions in each PVT cube. The recorded energy spectrum for 


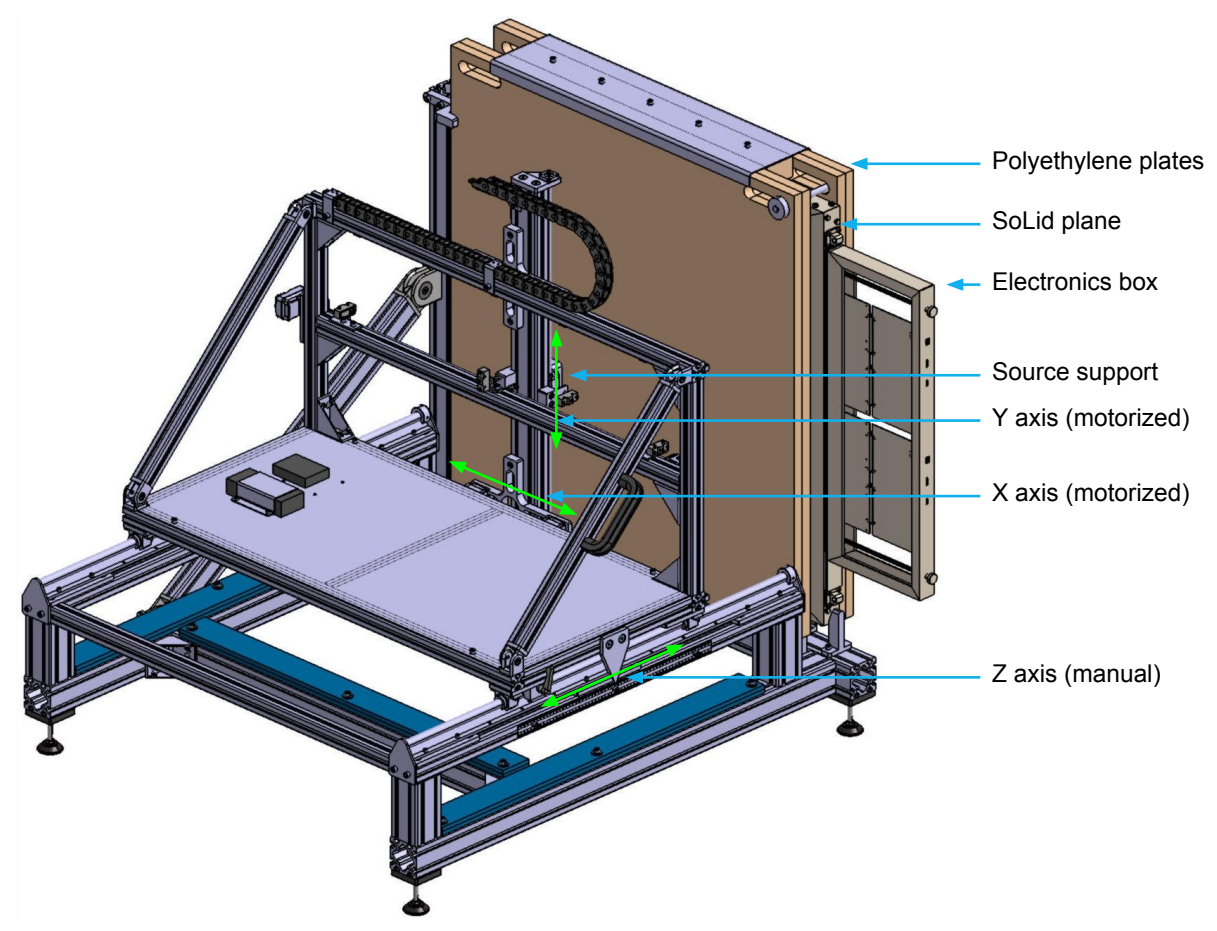

Figure 2. Illustration of the CALIPSO system for calibration of the SoLid planes, which operates in both neutron and gamma modes. The electronic box is placed on the right hand side of the planes. The CALIPSO robot provides sub-millimetre precision for accurate and consistent placement of the radioactive sources at any point in the XY plane.
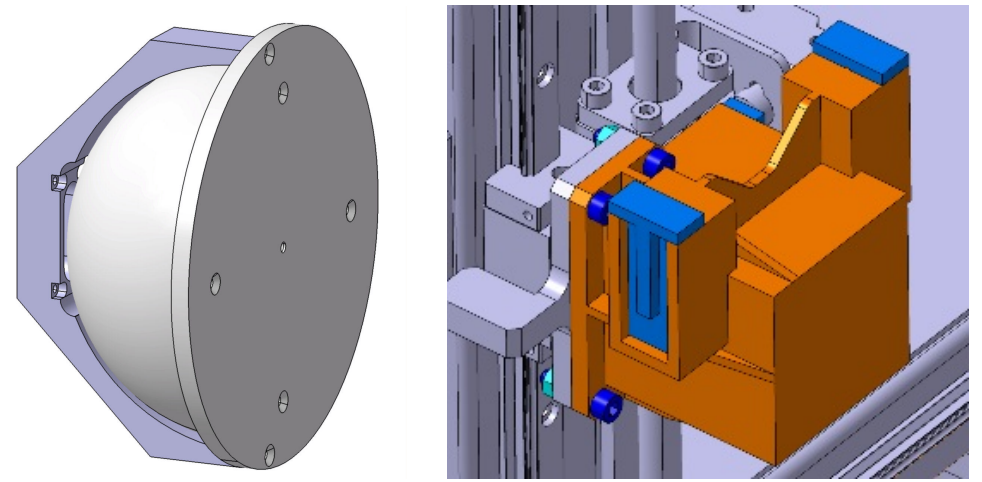

Figure 3. Left : Neutron collimator. Right : 3D external head for gamma calibration with ${ }^{22} \mathrm{Na}$ source.

each cube is then used to extract the light yield per cube using two approaches that are described in section 4.

Neutron response was studied using standard commercial AmBe and ${ }^{252} \mathrm{Cf}$ neutron sources. The activity reported by the manufacturer at the time of fabrication (November of 2016) of the sources are $37 \mathrm{MBq}$ and $37 \mathrm{kBq}$ for the $\mathrm{AmBe}$ and ${ }^{252} \mathrm{Cf}$ respectively ${ }^{1}$; however, the neutron yield is much lower as presented in table 1 . These sources underwent a dedicated calibration process

${ }^{1}$ For the AmBe source the quoted activity is the $\alpha$ activity of ${ }^{241} \mathrm{Am}$, and in the case of the ${ }^{252} \mathrm{Cf}$ the quoted activity includes $\alpha+$ fission rate decays. 


\begin{tabular}{|c|c|c|}
\hline Source & AmBe & ${ }^{252} \mathrm{Cf}$ \\
\hline Activity $(\mathrm{n} / \mathrm{s})$ & $1794 \pm 35$ & $3763 \pm 44$ \\
\hline $\mathrm{E}_{\text {max }}$ & $11 \mathrm{MeV}$ & $15 \mathrm{MeV}$ \\
\hline $\mathrm{E}_{\text {mean }}$ & $4.2 \mathrm{MeV}$ & $2.1 \mathrm{MeV}$ \\
\hline
\end{tabular}

Table 1. Neutron activity of sources used during the QA process as calibrated by the National Physical Laboratory (UK) on January of 2017. By December of 2017 when the QA was ending the estimated neutron activities were 1791.5 and $2993.2 \mathrm{n} / \mathrm{s}$ for the $\mathrm{AmBe}$ and ${ }^{252} \mathrm{Cf}$ respectively.
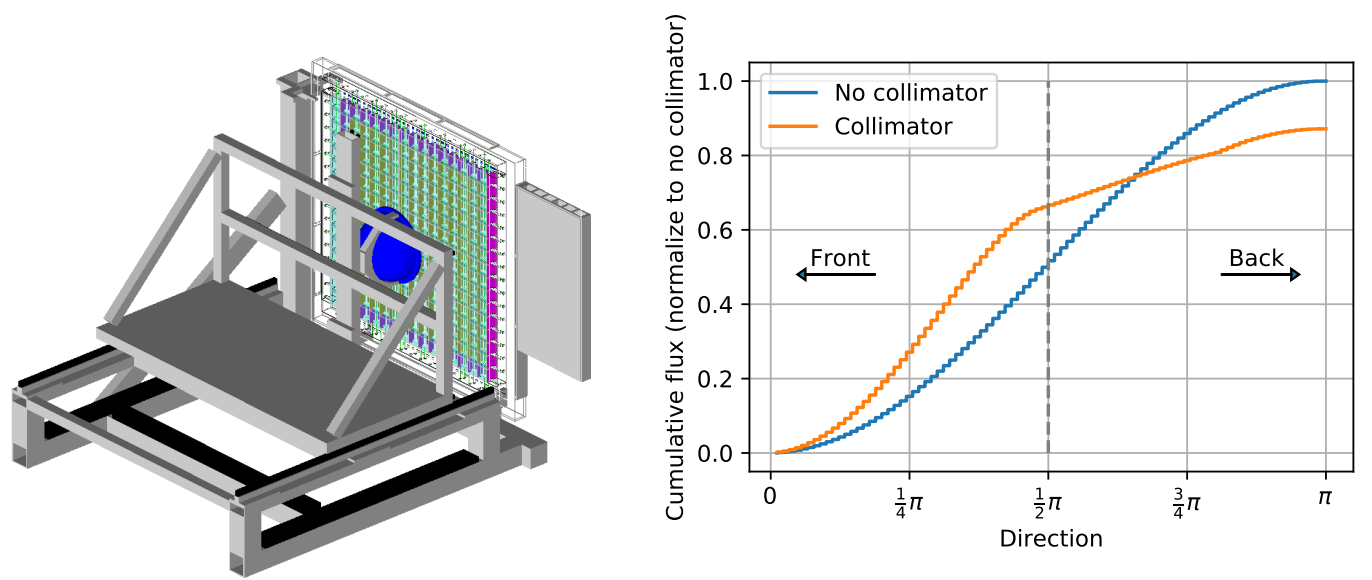

Figure 4. Left : CALIPSO GEANT4 Monte Carlo model in neutron mode. The neutron collimator can be observed in blue. Right : effect of the neutron collimator on the neutron flux as a function of the angle with respect to the orthogonal axis of the SoLid plane.

at the National Physical Laboratory (NPL) in the UK, where uncertainties in the neutron flux of $2 \%$ for the $\mathrm{AmBe}$ and $1 \%$ for the ${ }^{252} \mathrm{Cf}$ were determined. This neutron activity has been used as reference for the Monte-Carlo estimations. An activity correction was applied using the half life of the two isotopes of 432.6 years for AmBe and 2.645 years for ${ }^{252} \mathrm{Cf}$. The mean energies of the neutrons emitted by these sources are $4.2 \mathrm{MeV}$ for the $\mathrm{AmBe}$ and $2.1 \mathrm{MeV}$ for the ${ }^{252} \mathrm{Cf}$, as shown in table 1.

Fast neutrons emitted from the $\mathrm{AmBe}$ or ${ }^{252} \mathrm{Cf}$ sources have a low probability $(<5 \%)$ of being captured directly in the plane mounted on CALIPSO. Consequently the probability of seeing a neutron captured after scattering from the CALIPSO apparatus is significantly increased. These scattered neutrons can bias the neutron response. Therefore, a significant reduction in the frequency of fast neutron scatterings from CALIPSO, into the cubes being calibrated, had to be ensured. For this purpose, while in neutron mode, CALIPSO was augmented with two polyethylene plates of $5 \mathrm{~cm}$ on each side of the plane, as previously illustrated in figure 2 . These two moderating volumes induce two effects on the fast neutron population detected within the cubes under investigation. Firstly, by moderating and thermalising the neutrons coming directly from the source, they increase the probability of seeing a direct interaction in the plane. Secondly, the PE plates reduce the probability of seeing an indirect interaction from a fast neutron initially scattered from the CALIPSO structure.

In addition to the fast neutron reduction regime, a neutron collimator was designed to influence 

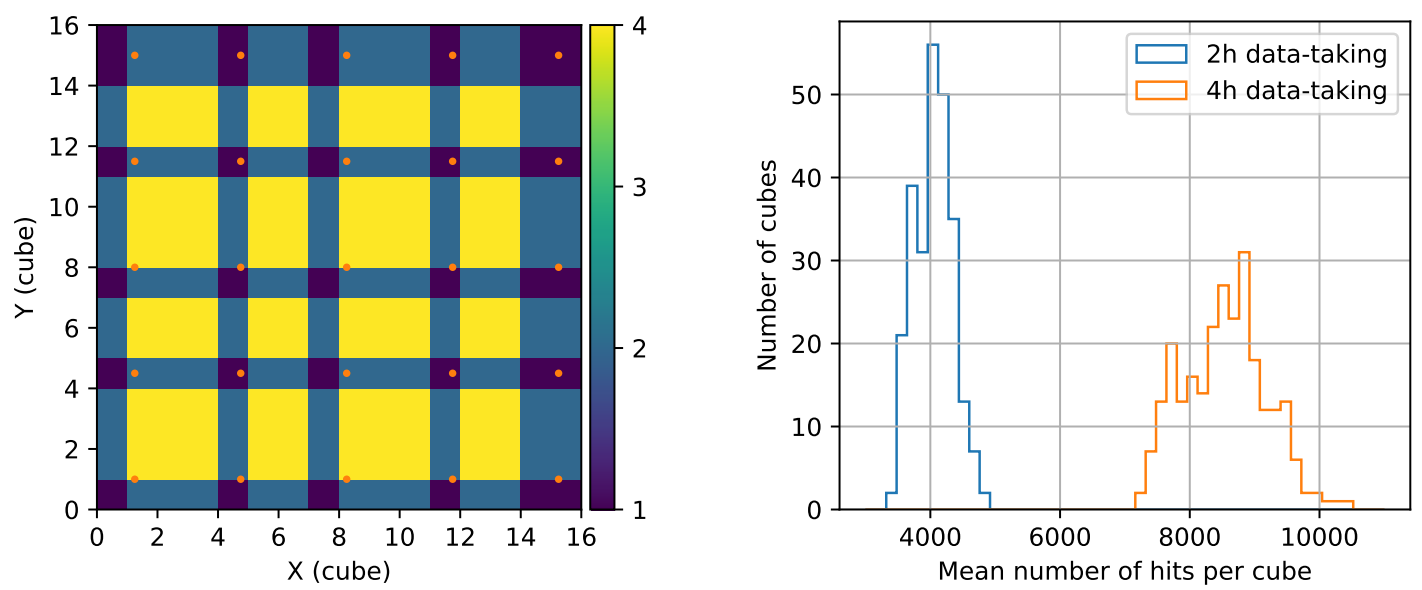

Figure 5. Left : Number of measurement points contributing to each cube after application of cuts. The neutron source is placed in 25 positions as illustrated with the orange dots. Only cubes close to the source are considered to the estimations. Right: Distribution of the mean number of hits per cube for 2 and 4 hours of data taking. A homogeneous distribution is achieved with the $25 \mathrm{XY}$ positions in each plane. The average number of hits recorded was $4051.1(\sigma=288.4)$ or $8542.9(\sigma=636.5)$ per cube for the 2 or 4 hours of data taking.

the directional neutron flux with respect to the cube under investigation.

The optimal collimator geometry was determined to increase the neutron detection rate by a factor of 1.4 due to both the forward collimation and from a less energetic flux (see figure 4). Thus, the number of neutrons entering the detector, after diffusion in the room, decreased by a factor of 5 to 10, depending of the position of the source. As an additional step in the analysis, only those cubes close to the source were used for calibration (see section 5); taking into account that the scattered neutrons are more likely detected in cubes further from the source position.

Combining these different actions, a Monte-Carlo study indicated that $\approx 98 \%$ of the captured neutrons are only scattered in the collimator-shielding-plane volume. In addition, this study showed that placing the source in this configuration for $25 \mathrm{XY}$ positions, as shown in figure 5, provides an homogeneous distribution of the NS events among the plane. This was important for the goal of accumulating at least 4000 neutron captures in each cube. Finally, given that the QA process was scheduled for six months, effects of mechanical shifting within the apparatus were reduced to a minimum. Consequently, this configuration could be maintained over a number of months, without the need for research personnel to closely monitor the immediate CALIPSO environment. Further details of the calibration process are expanded upon in section 5.

\section{Light Yield Measurement}

In order to assess the light yield (LY) of each SoLid voxel, a ${ }^{22} \mathrm{Na}$ gamma source was used, in conjunction with an external trigger. The external trigger consists of a PVT cube, read out by a small wavelength shifting fibre, coupled with an MPPC at each end. The system is contained within 


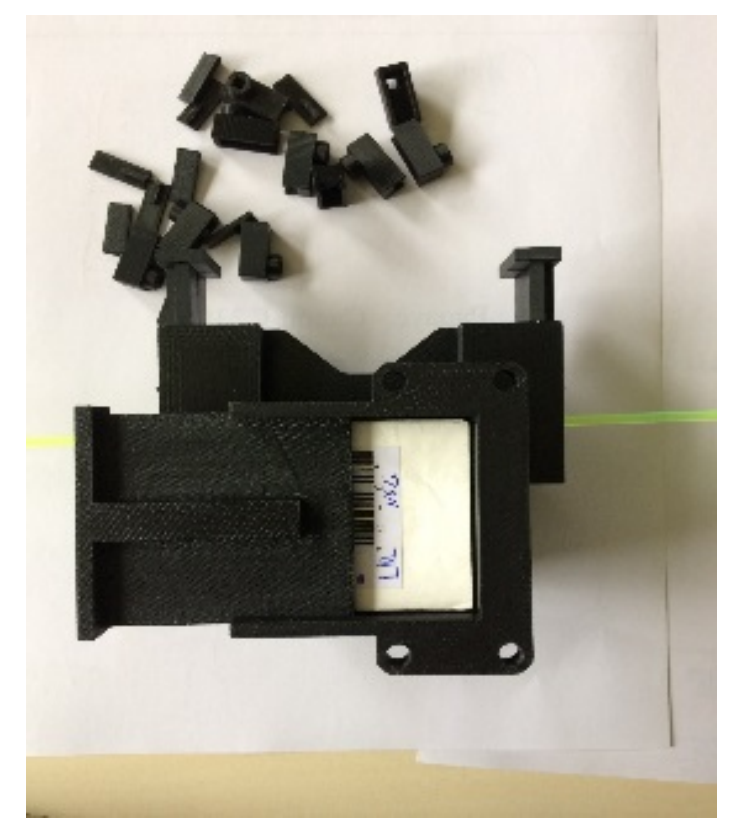

Figure 6. 3D printed external head for housing the external trigger assembly and the ${ }^{22} \mathrm{Na}$ source.

a 3D printed, externally mounting head; designed to combine the external trigger components, together with the ${ }^{22} \mathrm{Na}$ source as shown in figure 6.

${ }^{22} \mathrm{Na}$ decays via $\beta^{+}(90.3 \%)$ and via electronic capture $(9.64 \%)$ into ${ }^{22} \mathrm{Ne}$ as illustrated in figure 7. In almost all cases, ${ }^{22} \mathrm{Na}$ decays into the first excited state of ${ }^{22} \mathrm{Ne}$, which in turn decays to its ground state via the emission of a $1.27 \mathrm{MeV}$ gamma. Hence, in $90 \%$ of the ${ }^{22} \mathrm{Na}$ decays the emission of a positron in conjunction with a $1.27 \mathrm{MeV}$ gamma occurs; however, the $e^{+}$annihilates inside the source capsule, emitting two back-to-back $511 \mathrm{keV}$ gammas. These gammas are then used for the external trigger as illustrated in figure 7. If one of these gammas interacts in the external cube, we deem this a triggered event, and the full SoLid plane is read out. In this way, calibration samples with almost zero background can be collected, which allows an accurate calibration using the 1.27 $\mathrm{MeV}$ gamma, in conjunction with the $511 \mathrm{keV}$ gammas. It is important to note that the activity of the source and its uncertainty have no impact on the estimation of the light yield using this method. However, they will affect the signal to background ratio and time of exposure which can be tuned to accumulate the required statistics.

\subsection{Determining MPPC Operating Voltage}

Before taking calibration data, the correct operation of all 64 MPPCs, within each frame, must be verified. The validation process began with an initial non-equalised over-voltage data run. In a few cases the initial run identified unresponsive MPPCs which were replaced before continuing with the process. As the operating breakdown voltage for each MPPC is different, and requiring all the MPPCs to operate with an over voltage $(\mathrm{OV})$ of $1.5 \mathrm{~V}$, a voltage scan is required to determine the individual breakdown values. The voltage scan consisted of 25 runs using a fixed high voltage, close to the manufacturers nominal value in conjunction with a variable low voltage input. In these runs the gain of each MPPC was measured, which increased linearly with the OV. The 

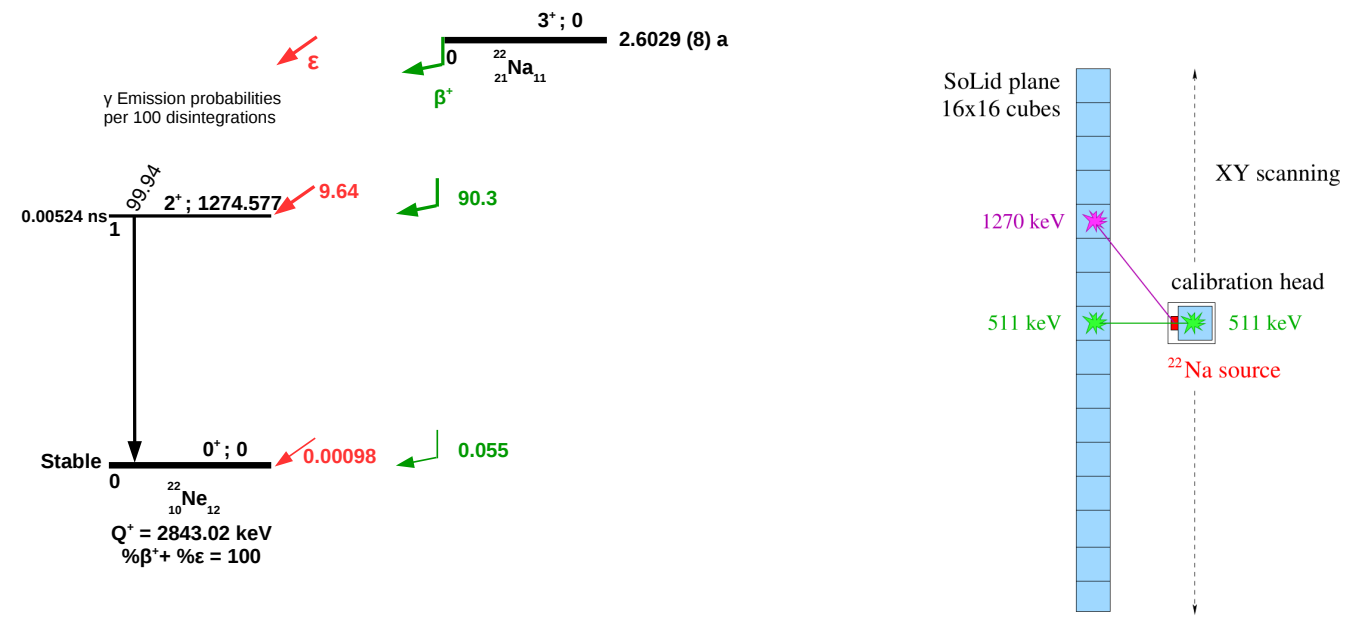

Figure 7. Left : Decay scheme of a ${ }^{22} \mathrm{Na}$ source. In about $90 \%$ of cases it decays via $\beta+$ emission to the first excited state of ${ }^{22} \mathrm{Ne}$, which in turn goes into its ground state through the emission of a $1.27 \mathrm{MeV}$ gamma. Right : Representation of the external trigger system using the $511 \mathrm{keV}$ gammas from the ${ }^{22} \mathrm{Na}$ source.

individual breakdown voltages were identified by fitting the resulting gain values as a function of the varying voltage, and extrapolating to a gain of zero. For an $\mathrm{OV}$ of $1.5 \mathrm{~V}$, a gain of about 22 Analogue-to-Digital Conversion units (ADC) per pixel avalanche was determined. Uncertainties in the estimation of the breakdown voltage, and the variation in gain response with voltage between the MPPCs, translated into the operational gains varying from channel to channel by about $3 \%$. This methodology was further refined during calibration runs after installation at BR2 and achieved an equalisation variance at the $1 \%$ level.

\subsection{Cube Light Yield and Signal Reconstruction}

An additional goal of the quality assurance process was to identify possible defective voxels in the SoLid planes. This was carried out by calculating the light yield in each voxel from the total amount of light collected in the 4 MPPCs associated with each cube. Variations in gain from channel to channel need to be taken into account before summing the signals of the 4 sensors, as each MPPC has a slightly different breakdown response. Gains for the individual voxels are recalculated by identifying the PA values in the ${ }^{22} \mathrm{Na}$ energy spectra; each PA distribution was fitted with a Gaussian function as shown in figure 8 to obtain the positions of the PA peaks. A linear fit of the PA peaks has a slope corresponding to the gain of each channel. The parameters of the fit function were tuned to operate automatically with the CALIPSO data.

Figure 8 shows the detected amplitude spectrum $A$, in ADCs for a channel using the ${ }^{22} \mathrm{Na}$ source. This spectrum is thus used to adjust the gains $g$. Once the gain is determined, it is used to make the conversion from ADC counts to PA. The first peak in figure 8 (Left) corresponds to the 6th PA peak, and not the 1 PA associated peak. This "shift" is caused by a zero suppression (ZS) threshold set to 5 PA being used during the data taking in order to reduce the data rate. This reduction in data rate is needed since the QA data taking is performed at standard room temperature $\left(\sim 25^{\circ}\right)$, 

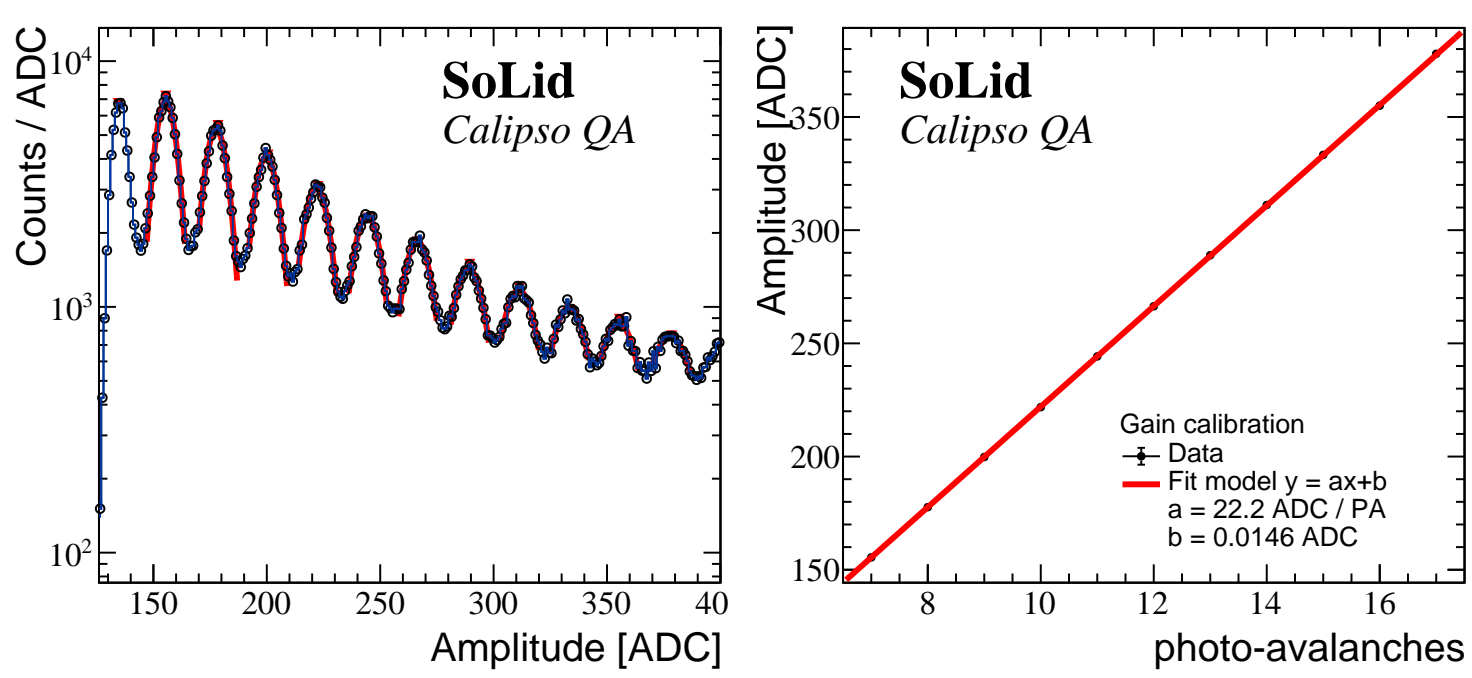

Figure 8. Left : Detected spectrum in an MPPC using the ${ }^{22} \mathrm{Na}$ source, the PA arrival peaks can be clearly identified. The first peak that appears in the spectrum corresponds to the sixth PA, not the first PA. The previous peaks are excluded in the data by setting a zero suppression threshold of 5 PA during the initial QA campaign.

Right : Linear fit of the PA peaks, where the slope corresponds to the gain. In this particular case the gain, $g$, is calculated at $22.2 \pm 0.01 \mathrm{ADC}$ per PA. The intersection of the curve for $0 \mathrm{PA}$ is $0.01 \pm 0.07 \mathrm{ADC}$, in very good agreement with a linear response.

at which the dark rate of peaks below $5 \mathrm{PA}$ is unacceptably high and dominates the data taken. The SoLid detector will operate at around $10{ }^{\circ} \mathrm{C}$, with data taking utilising a ZS threshold of 1.5 PA. This ZS is driven by the data rate, a ZS value at $0.5 \mathrm{PA}$ will remove the pedestal contribution, whilst retaining all SiPM signals, resulting in a waveform compression factor of around 50. Increasing the threshold further to 1.5 PA can provide another order of magnitude of waveform compression, at the expense of removing the single PA signals [24].

To reconstruct the total amount of light produced in a given cube, the total ${ }^{22} \mathrm{Na}$ spectrum per cube must be computed. To effect this, coincidences are sought between the two vertical and the two horizontal sensors, coupled to the 4 fibres going through each cube. Thus, the total amplitude per cube $A_{i j}$ in PA is defined as:

$$
A_{i j}=\frac{A_{i}^{t}}{g_{i}^{t}}+\frac{A_{i}^{b}}{g_{i}^{b}}+\frac{A_{j}^{l}}{g_{j}^{l}}+\frac{A_{j}^{r}}{g_{j}^{r}}
$$

with $t, b, l, r$ for the position of the sensors on the top, bottom, left and right sides of the plane respectively, and $i, j$ for the cube co-ordinates in the plane.

Gammas from the ${ }^{22} \mathrm{Na}$ source $(511 \mathrm{keV}$ and $1270 \mathrm{keV}$ ) interact in the PVT mostly through Compton scattering. In addition, given the granularity of the detector planes, only a fraction of the total gamma energy is deposited within each PVT cube. Consequently no narrow photo-peak can be reconstructed within individual cubes. The light yield must therefore be derived from a more complicated distribution, and two approaches were employed to this end. The first method 
consists of fitting the Compton edge profile of the spectrum by an analytical function and the result compared to the predicted value. The second method compares the measured energy spectrum to a Geant4 simulated sample varying the light yield and energy resolution. In the next two sections these methods are discussed in more detail.

\subsection{Compton Edge Analytical Fit}

For a given cube undergoing calibration, we assume that a $1.27 \mathrm{MeV}$ gamma interacts within the cube only via Compton scattering and, that the gamma only scatters once per cube. In this instance, the distribution of the true energy deposits of scattered electrons is defined according to the Klein-Nishina cross-section, $\sigma_{c}$ [26, 27]:

$$
\frac{d \sigma_{c}}{d T}=\frac{\pi r_{e}^{2}}{m_{e} c^{2} \alpha^{2}}\left(2+\left(\frac{T}{E_{0}-T}\right)^{2}\left(\frac{1}{\alpha^{2}}+\frac{E_{0}-T}{E_{0}}-\frac{2}{\alpha}\left(\frac{E_{0}-T}{T}\right)\right)\right)
$$

where $T$ represents the kinetic energy of scattered electrons, $\alpha=E_{0} / m_{e} c^{2}, m_{e}$ the electron mass and $E_{0}$ the initial energy of the incident photon. This cross section is peaked for energies approaching the kinematical limit for the energy transferred to the scattered electron, and displays an abrupt fall to zero above this energy. This Compton Edge (CE) is the strongest feature of this distribution and is used to determine the light yield. Determining the CE position in the distribution of PAs, can be translated into a light yield since the theoretical CE is well known. The theoretical CE is computed using an angle of deflection, $\theta$, of the incident gamma of 180 degrees. This leads to the following equation:

$$
E_{C E}=E_{T}(\max )=E_{0}\left(1-\frac{1}{1+\frac{2 E_{0}}{m_{e} c^{2}}}\right)
$$

wich only depends of the initial energy of the incident gamma $E_{0}$ and the electron mass $m_{e}$. For gammas of $511 \mathrm{keV}$ and $1270 \mathrm{keV}$ the Compton edges are $341 \mathrm{keV}$ and $1057 \mathrm{keV}$ respectively.

Experimentally the Compton edge is smeared according to the energy resolution of the detector. The CE value can be identified by using the shape of the energy spectrum; then fitting this spectrum profile according to equation 4.2, convoluted with a Gaussian function, in order to account for a stochastic energy resolution.

The resulting function can be written as:

$$
f(x)=\int_{0}^{E_{C E}} \frac{d \sigma_{c}}{d T}(T) \frac{1}{\sqrt{2 \pi} \sigma_{0} \sqrt{T}} e^{-0.5 \frac{(x-T)^{2}}{\sigma_{0}^{2} T}} d T
$$

And the necessary normalisation term to obtain a probability density function (pdf) is given by:

$$
\int_{-\infty}^{\infty} f(x) d x=\int_{0}^{E_{C E}} \frac{d \sigma_{c}}{d T} d T
$$

where $E_{C E}$ and $\sigma_{0}$ are respectively the Compton edge and the energy resolution.

Figure 9 shows the energy spectrum for a calibration sample in a SoLid cube. The Compton edge profile has been fitted using the $p d f$ defined in equation 4.5. The Compton edge is estimated in 85.8 PA respectively, which can be translated in a light yield of $81.2 \mathrm{PA} / \mathrm{MeV} / \mathrm{Cube}$. On the 


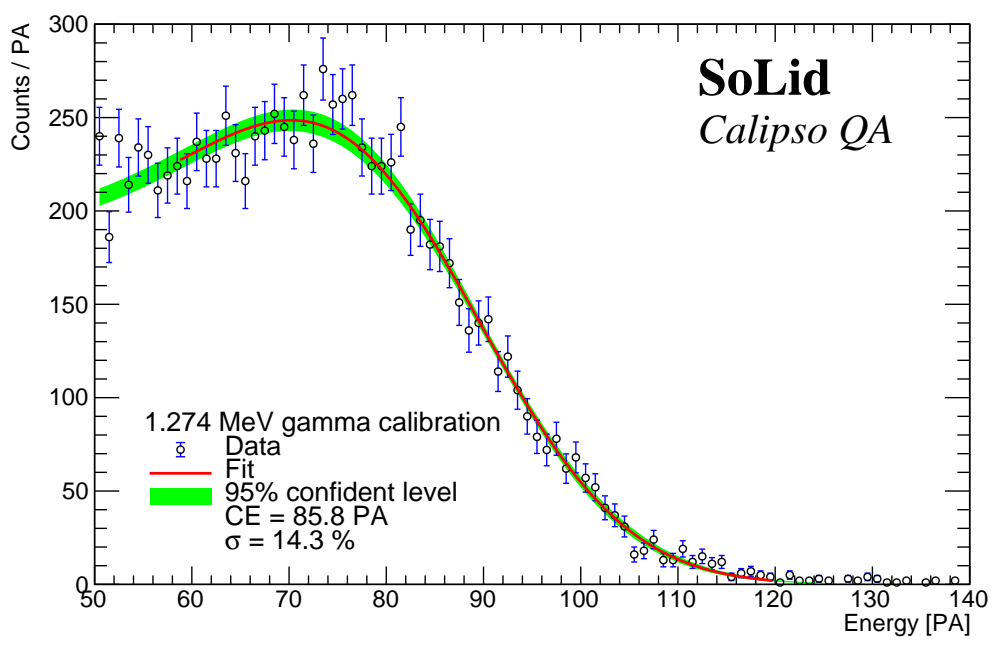

Figure 9. Compton edge profile for a calibration sample in a SoLid cube using a ${ }^{22} \mathrm{Na}$ gamma source. The spectrum is dominated at high energy by the deposits of the $1.27 \mathrm{MeV}$ gamma. The Compton edge is obtained by fitting the distribution with the $p d f$ defined in equation 4.5 .

other hand, the energy resolution is estimated to be about $14 \%$, inline with the SoLid physics requirements. The accuracy of this fit has been evaluated with the Monte-Carlo. It was found that in the case of the $1.27 \mathrm{MeV}$ gamma from the ${ }^{22} \mathrm{Na}$ source, the estimation of the $\mathrm{CE}$ is biased by about $+3.5 \%$. This bias can be explained by cases of multiple scattering in the same cube, rather than the assumed single scattering, leaving a deposited energy per cube higher than $E_{C E}$.

\subsection{Kolmogorov-Smirnov Test}

A second light yield estimation is obtained from comparing a GEANT4 simulated ${ }^{22} \mathrm{Na}$ energy spectrum with the observed sample using a Kolmogorov-Smirnov (K-S) test. The true deposited energy in each cube is used to build a set of spectra with different energy resolutions, varying from $5 \%$ to $20 \%$. Each spectrum is then compared to the calibration sample for each cube, varying the light yield from 50 to 120 PA. The light yield is taken from the point of best agreement between the spectra of the calibration sample and the prediction. At this point the K-S test maximises as is shown in Figure 10.

Figure 10 shows the K-S test results for a specific cube; where the K-S test maximises for a LY of $83 \mathrm{PA} / \mathrm{MeV}$, as shown at the bottom of figure 10 . For values where the data and the Monte-Carlo are not compatible, the K-S test returns zeroed values. Varying the section of the spectrum, the binning, and the number of steps used to maximise the K-S test, a systematic error of about $2 \%$ was estimated for this method.

Finally the two methods of CE analytical fit and K-S test were compared in order to validate the procedure and provide an estimation of the systematic uncertainties. Both approaches assume that the convolution product is correct, which means a Gaussian behaviour of the energy resolution at 1 $\mathrm{MeV}$, and that the reconstruction efficiency is flat in E. Since only the region around $1 \mathrm{MeV}$ is used, no sizeable effect for introduced errors has been found when using the MC. All other sources of systematic uncertainties are reasonably assumed to be measured by the difference between the two 

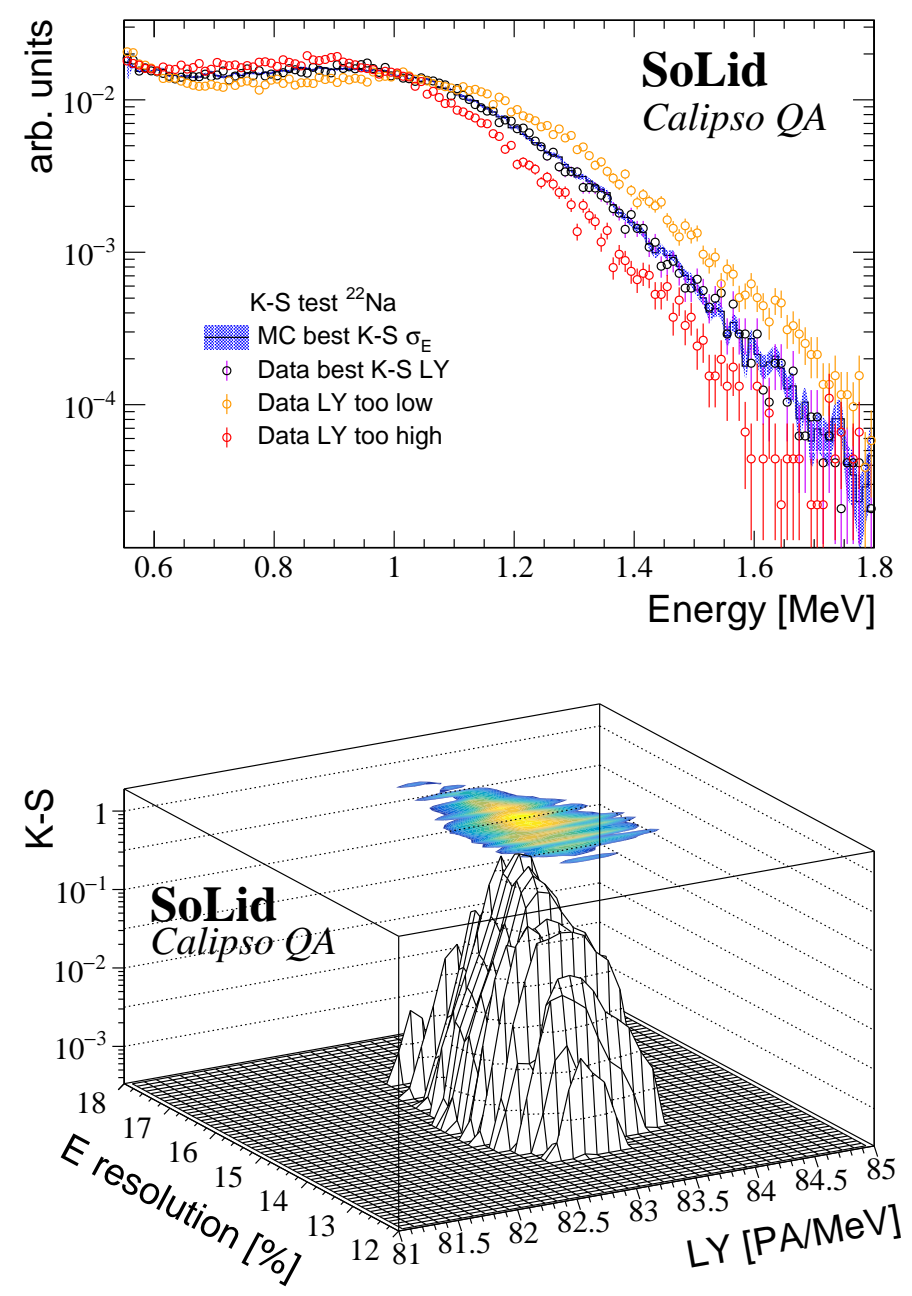

Figure 10. (Top) Data compared to MC for different values of LY. Violet corresponds to a LY value where the K-S test is maximised, while the red and yellow show two cases where the LY is found to be too high and too low respectively. (Bottom) Distribution of the K-S test values in the parameter space of energy resolution from MC and light yield from the data. The K-S test takes values of 0 when the data is not compatible with the predicted spectrum, and take positive values when agreement is found.

approaches because they are based on completely different assumptions; the analytical fit supposes that there is only one single scattering per cube per event, while the K-S test assumes that the Geant4 MC is correct.

A very good agreement was found between the values of LY obtained using the K-S test and the method of fitting with an analytical Compton $p d f$ after bias correction as shown in figure 11. The difference between moth methods remains at less than $2 \%$. The tails on the sides of figure 11 corresponds to cubes with one problematic channel, where the accuracy of the analytical fit is less good. Since the objective of the QA process was only verify the minimal requirements to achieve the SoLid goals, a $2 \%$ of systematic uncertainty in the energy scale was sufficient at this stage. The K-S test method was deemed more rigorous when looking to automate the procedure for the 12800 


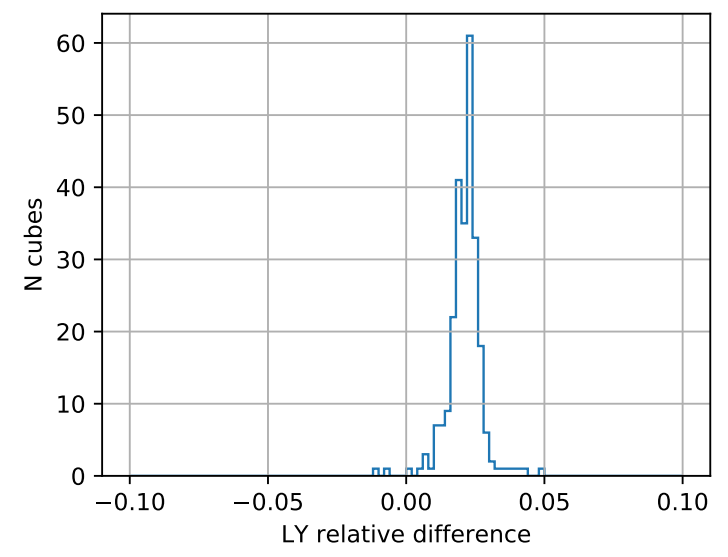

Figure 11. Relative difference in the light yield results for frame 10 using two methods, analytical fit and K-S test. A very good agreement can be observed. A difference of about $2 \%$ can be observed, which can be due to the cuts used for the sample selection for the analytical fit.

cubes, and so this test was used during the QA process prior to the detector construction.

Calibration samples for all 50 frames were collected; as such the LY of each SoLid cube was evaluated prior to detector assembly. For the in-situ calibration at BR2, a combination of the 2 approaches are continually being used, providing a good control of systematic uncertainties.

\subsection{Construction Adjustments}

Evaluating the light yield for each cube provides a good tool for identification of defective components during the construction of the SoLid planes. For example, figure 12 shows the results of the measured light yield for plane 13, using the K-S method. Looking at this frame, a number of effects can be observed. Firstly, it shows that the cubes placed at the border of the frame have a higher LY than those in the centre. This effect is expected if we consider the attenuation of the light within the wavelength shifting fibres used for the readout of the signals, which is estimated to be of the order of $\sim 100 \mathrm{~cm}$, which is comparable to the width of one SoLid plane. Secondly, column 11 shows a light yield that is more than $10 \%$ lower compared to the neighbouring cubes. This low light yield can not be explained in terms of attenuation length, and in cases such as these corrective actions were undertaken.

In most of these interventions of the row/column showing a low LY, it was observed that the coupling between one of the fibres and its MPPC or mirror were partially or completely loose. These cases were fixed by either adjusting the connector interface of the MPPC or mirror, or by re-adhering the connector to the aluminium support frame used to mount the planes.

\subsection{Light Yield Results}

This initial calibration with CALIPSO served not only for quality assurance purposes, but also to obtain a first estimation of the light yield. Consequently a light yield of $83 \mathrm{PA} / \mathrm{MeV} / \mathrm{Cube}$ before MPPC crosstalk subtraction was measured, as can be observed in figure 13. Because of time constraints it was not possible to take dedicated crosstalk measurements with the CALIPSO system. 


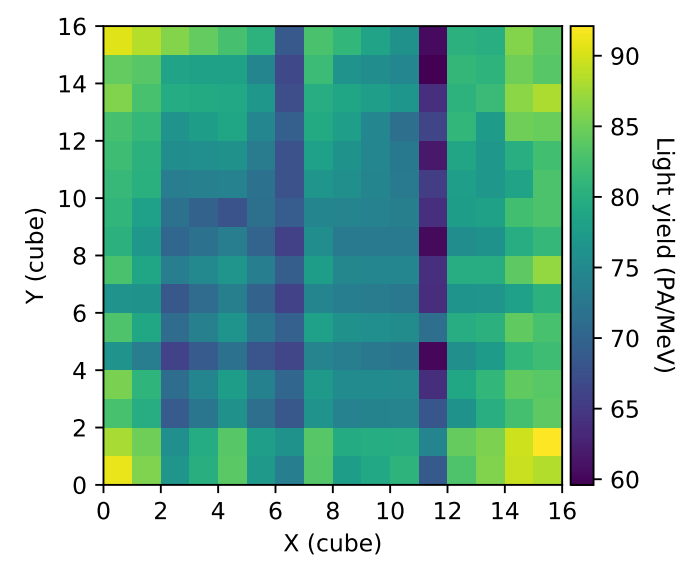

Figure 12. Estimated LY in frame 13 before MPPC crosstalk correction. Column 11 shows a deficit in the LY, which was identified as a bad coupling between the fibre and the MPPC located in the top of the frame.

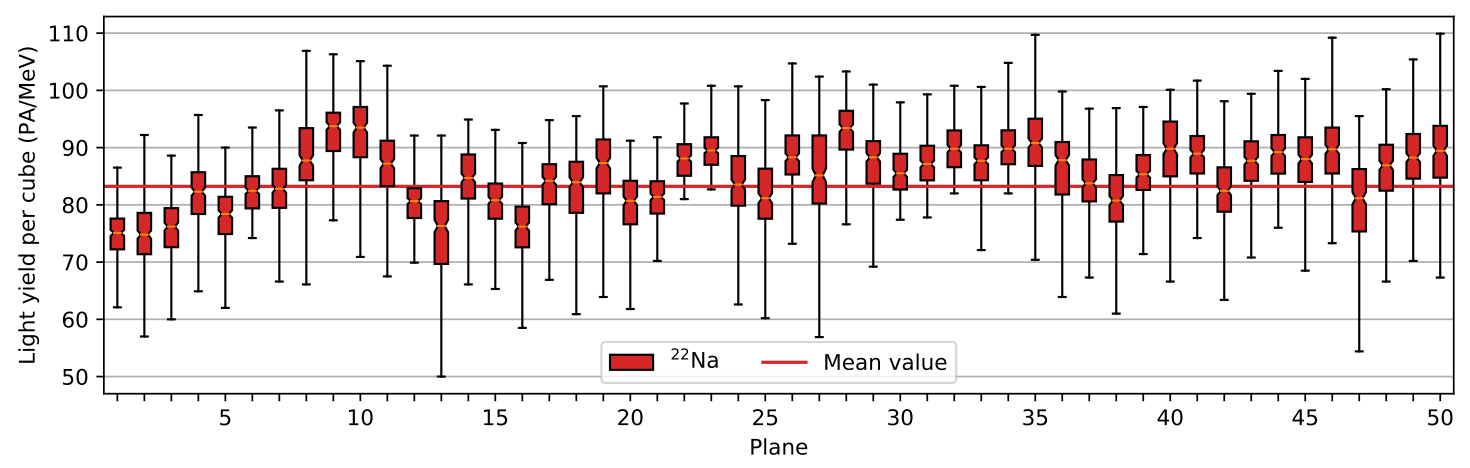

Figure 13. CANDLE plot for the light yield of the 50 planes of the SoLid detector obtained with a ${ }^{22} \mathrm{Na}$ gamma source. An average of $83 \mathrm{PA} / \mathrm{MeV} / \mathrm{Cube}$ was found without MPPC cross-talk subtraction, which is estimated to be around $17 \%$. Orange line represents the mean value of each plane, while filled boxes represent cubes between the first and the third quartiles (50\% of the data points). Black lines represent cubes below and above respectively the first and third quartiles.

However, the MPPC crosstalk $^{2}$ has been estimated through other means at $\approx 17 \%$ for an OV of $1.5 \mathrm{~V}$ [22]. Therefore the results presented in this paper do not include correction for crosstalk. Nevertheless, assuming a MPPC crosstalk of $17 \%$, the final light yield is expected to be larger than $70 \mathrm{PA} / \mathrm{MeV} / \mathrm{Cube}$, inline with the SoLid physics requirements.

A light yield of about $70 \mathrm{PA} / \mathrm{MeV}$ allows to reach an energy resolution of around $12 \%$. Moreover it could be improved, since the CALIPSO calibration data was taken at an OV of $1.5 \mathrm{~V}$, while the full detector will operate at BR2 at an $\mathrm{OV}$ of $1.8 \mathrm{~V}$, increasing the photon detection efficiency by about $20 \%$.

${ }^{2}$ This crosstalk is defined as the probability that an avalanching pixel will cause an avalanche in a second pixel. The process happens instantaneously an as a consequence, single incident photons may occasionally generate signals equivalent to 2 or 3 photons, or even higher depending on the OV. 


\section{Neutron Detection Efficiency}

The IBD detection efficiency is dominated by the neutron detection efficiency; therefore the neutron detection parameter needs to be optimised and accurately determined.

The neutron detection efficiency can be defined as :

$$
\epsilon_{\text {det }}=\epsilon_{\text {capt }} \times \epsilon_{\text {trig }} \times \epsilon_{P I D}
$$

where $\epsilon_{\text {capt }}$ is the probability for a neutron to be captured by the ${ }^{6} \mathrm{Li}$ in the ${ }^{6} \mathrm{LiF}: \mathrm{ZnS}$ layers, $\epsilon_{\text {trig }}$ corresponds to the probability of triggering the read-out on a NS signal and $\epsilon_{P I D}$ is the offline Particle Identification (PID) efficiency. The last two variables can be factorised as the reconstruction efficiency $\epsilon_{\text {reco }}=\epsilon_{\text {trig }} \times \epsilon_{P I D}$. The neutron capture efficiency $\epsilon_{\text {cap }}$ is estimated from a MonteCarlo study and depends collectively on; the neutron emission point, the energy of the neutron, the detector geometry, the $\mathrm{H}$ content and, the ${ }^{6} \mathrm{Li}$ content of the ${ }^{6} \mathrm{LiF}: \mathrm{ZnS}$ layers. Using the CALIPSO based Monte-Carlo configuration, a mean capture efficiency of $\epsilon_{\text {capt }} \approx 8.7 \%$ has been estimated, with typical variance from $10.6 \%$ for a source in the plane centre and $6.5 \%$ in the plane corner.

The approach of the QA campaign has been to relatively compare each plane, considering that every plane should provide a similar capture efficiency. Once environmental effects are minimised, a like-for-like comparison can be made on a positional basis. From this the relative efficiency $\epsilon_{r e l}$ of each cube for all given positions is ascertained, while retaining sensitivity to a large $\epsilon_{\text {capt }}$ variation across a plane. With these positional comparisons; homogeneity, edge effects and other performance related factors can be determined. The absolute efficiency of the entire detector depends on the complete detector geometry and will be determined in-situ after construction is complete at BR2.

\subsection{Nuclear Signal Reconstruction}

The reconstruction chain from trigger to offline analysis, was also commissioned during the QA process. In order to reach a high neutron detection efficiency, the Pulse Shape Discrimination (PSD) is managed in two steps, that are described in the following sections.

\subsubsection{Neutron Trigger}

The neutron trigger is designed to maximise neutron detection efficiency, even if it is at the expense of the trigger purity. However, a high trigger purity can also be achieved by decreasing the neutron detection efficiency. For instance, increasing the trigger purity from $40 \%$ to $80 \%$ decreases the neutron reconstruction efficiency from $70 \%$ to 57\% [24]. The first level of PSD is implemented in the trigger firmware. This requirement can be reached with a simple Peaks over Threshold (PoT) algorithm. This PoT algorithm can be implemented since the time constants of PVT and ZnS scintillators are completely different. An electronic scintillation from the PVT, lasting $\approx 20 \mathrm{~ns}$, is composed of 1 sharp peak; while a nuclear scintillation from the $\mathrm{ZnS}$, lasting $\approx 10 \mu \mathrm{s}$, is composed of many individual peaks, because of the extended scintillation within the ZnS. Using this simple PoT approach, the trigger decision is taken if a certain number of peaks above a given threshold is reached, within a rolling time window (see figure 14). For the QA campaign, the priority was for a high trigger purity i.e. the number of actual neutrons from the PoT trigger; in this case a higher threshold was demanded in the trigger requirements (PoT). In this way a trigger purity exceeding 

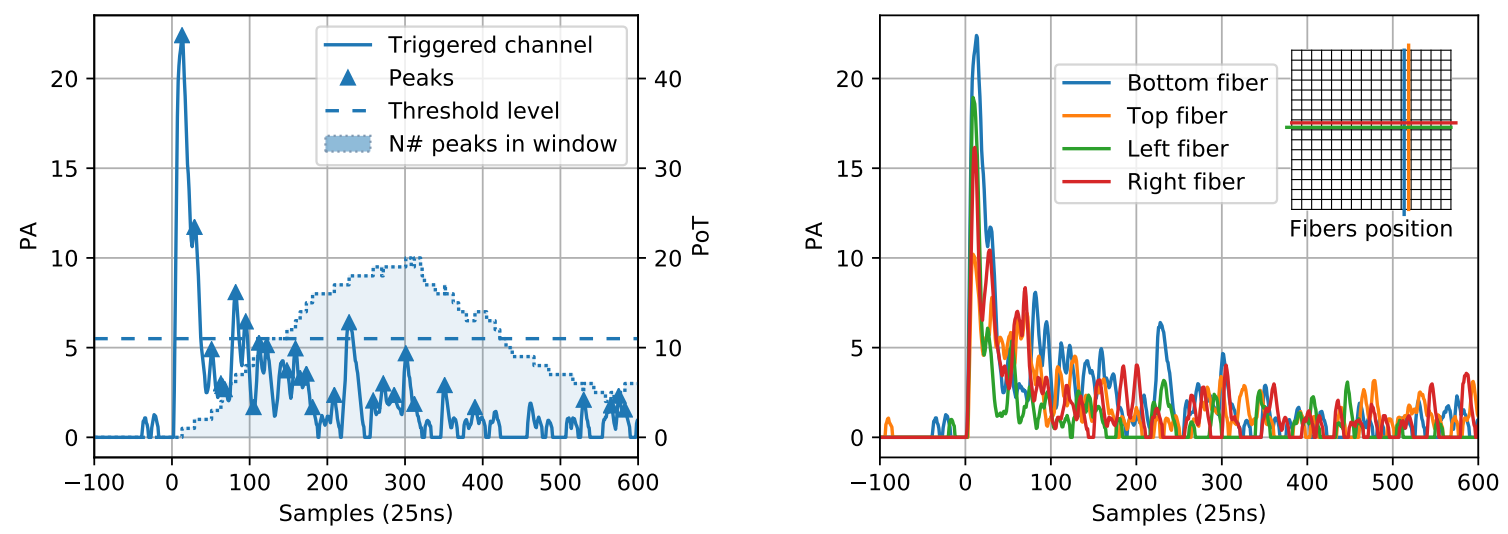

Figure 14. Left: Triggered NS signal. Peaks (i.e. samples above 1.5 PA and above its two neighbours) are represented by triangles. The filled area represents the number of peaks in the previous 256 sample window (Peak over Threshold or PoT). Read-out is triggered when the PoT value is above 11 Peaks (dashed line on the figure).

Right : Reconstructed NS event, composed from 4 fibres. Positions of the fibres in the plane are shown in the upper right insert. From the intersection point, the voxel containing the interaction can be determined.

$99 \%$ has been achieved. However, this decreased the detection efficiency. For the standard data taking in physics mode at BR2, the trigger purity is expected to be $\sim 20 \%$. This value was initially estimated in order to achieve a neutron reconstruction efficiency of around $80 \%$. Further optimisations increased trigger purity to about $37 \%$ keeping a neutron reconstruction efficiency of about $80 \%$ [24].

\subsubsection{Neutron Particle Identification}

The information from the online neutron trigger is used to initialise the parameters for offline analysis of the NS candidates. By finding the combination of 4 channels that maximise the number of peaks over threshold in the trigger window, it is possible to reconstruct the interacting cube position within the plane (see figure 14). Combining the information from these fibres, one can compute the amplitude and integral of the NS signal. From these two parameters, an NS signal is placed on the "Amplitude vs Integral over Amplitude" (IonA) parameter space, as shown in figure 15. This results in an efficient and pure separation of ES and NS signals. It can be noticed that the main ES contamination in NS candidates is from high amplitude signals, more likely corresponding to muons crossing the plane.

\subsection{Neutron Detection Efficiency Estimation}

The goal of the relative efficiency measurement was to compare the number of NS-like events for a given voxel to the mean number of NS-like events seen throughout all planes, on a positional basis.

Thus, the number of NS-like events for a given voxel with coordinates $\mathrm{x}, \mathrm{y}$ in the plane $\mathrm{i}$ $(N(x, y, i))$ can be measured, and the relative efficiency, $\epsilon_{r e l}(x, y, i)$, can be defined as:

$$
\epsilon_{r e l}(x, y, i)=\frac{N(x, y, i)}{\bar{N}(x, y)}
$$




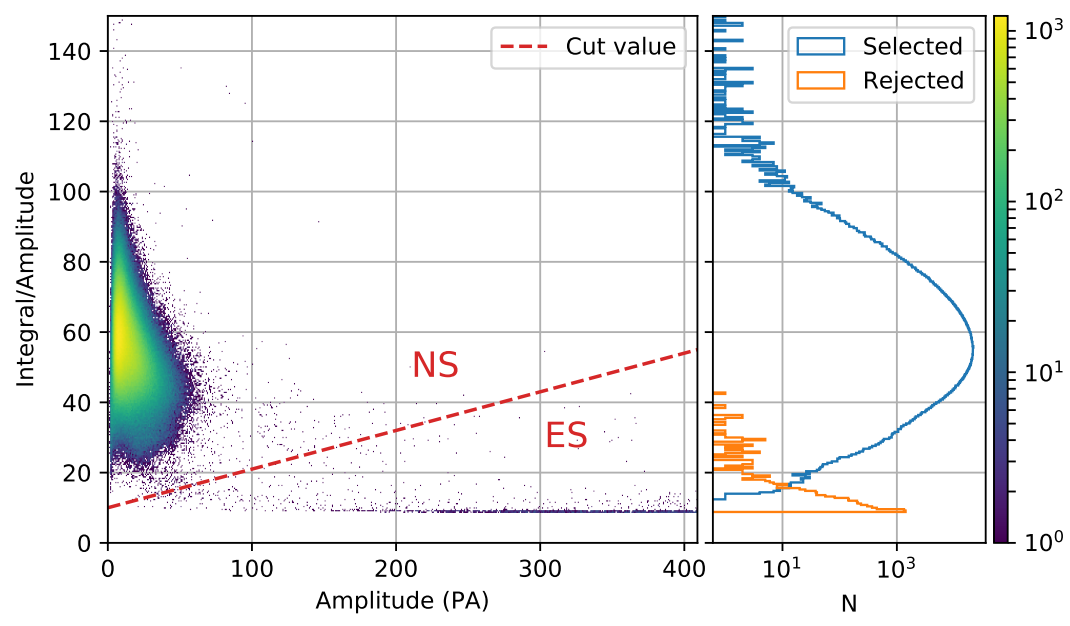

Figure 15. Integral over Amplitude versus Amplitude, in Photo-Avalanches(PA) for reconstructed NS candidates from data taken with a ${ }^{252} \mathrm{Cf}$ source on plane 50 . The red dashed line shows the cut used for the Particle Identification. The right panel presents the projection on the Integral over Amplitude axis for selected and rejected events.

with :

$$
\bar{N}(x, y)=\frac{1}{50} \sum_{i=1}^{50} N(x, y, i)
$$

Finally, the 25 measurements for each plane are merged in order to obtain the total number of NS-like events for each of the 256 voxels composing the plane, as shown in figure 5. However, two effects need to be taken into account to execute this operation:

1. In order to maximally reduce environmental effects, only cubes close to the source are added to the global measurement.

2. The exposure time for each of the 25 calibration positions will have some degree of variance.

The first effect is taken into account during the merging process, by only selecting cubes within a given distance for $\mathrm{X}$ and $\mathrm{Y}$ of \pm 3 cubes, resulting in a square centred around the source. The total number of contributing points for each cube can be seen in figure 5 .

The number of NS-like events is homogeneous across any given plane, even if only one point of measurement contributes from each of the voxels at the edges of the plane. Therefore an homogeneous statistical uncertainty over the whole plane is reached, with minor edge effects on each plane. This edge effect can be explained by the presence of the polyethylene reflector between the cubes and the aluminium frame. Nevertheless, due to the second effect of exposure time variance, it is not possible to directly compare these merged NS-like maps. Hence, for each plane, an expected NS-like map is built for this purpose. Thus, for a given plane $i$, the NS-like map is based on the average rate observed at each measurement point over all the planes. Finally the map is normalised to the time of measurement for the plane $i$. 


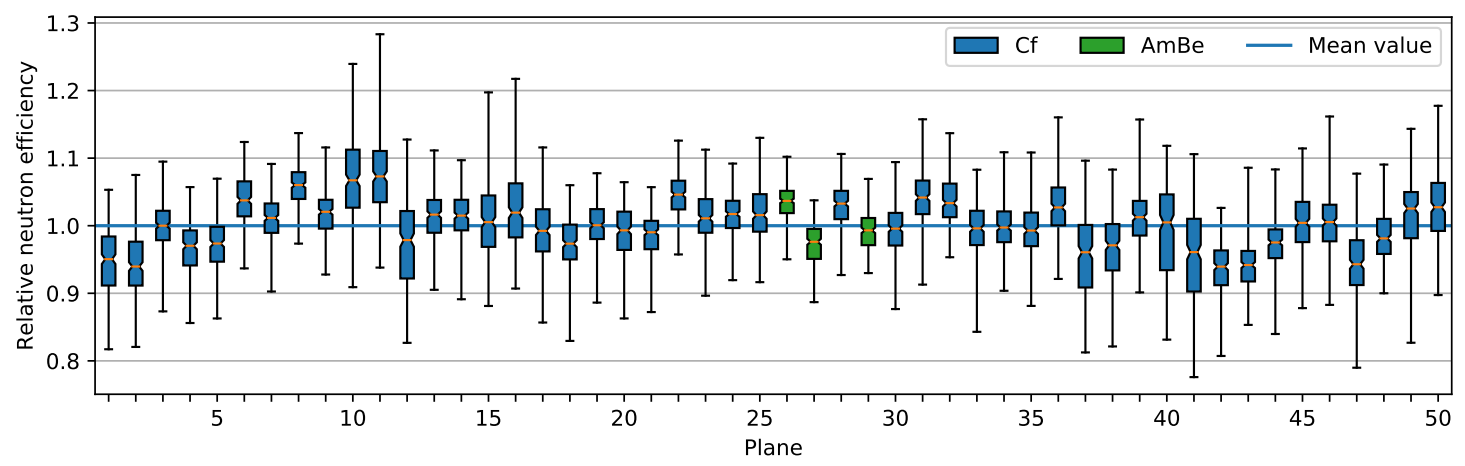

Figure 16. CANDLE plots for the relative neutron efficiency for the 50 planes of the SoLid detector. Orange line represents the mean value for each plane. Filled boxes represent cubes between the first and the third quartiles (50\% of the data points). Black lines represent cubes below and above respectively the first and third quartiles. Results are separated in two sets: blue corresponds to planes tested with ${ }^{252} \mathrm{Cf}$, green with AmBe.

Thus, $\epsilon_{r e l}$ for the plane $i$ can be determined as the ratio of the merged rate for $i$ divided by the mean rate observed in all the planes. Results for $\epsilon_{r e l}$ are presented in figure 16. A $\sigma$ of 5\% is found, exceeding initial requirements of having a dispersion smaller than $10 \%$.

From these measurements, it is possible to extract an absolute $\epsilon_{\text {reco }}$, by comparison to Monte Carlo expectations for the 25 calibrations points. However, the accuracy in the simulation is not sufficient to do this exercise per cube, but it can be done to determine an overall efficiency. This comparison provided an $\epsilon_{\text {reco }} \approx 68.7 \%$, in-line with the SoLid physics requirements. With a neutron purity $\epsilon_{P I D}$ above $99 \%$, which is mainly driven by $\epsilon_{\text {trig. }}$. Further optimisations will be done in-situ at BR2, where the trigger will be tuned to find the best performance metric and achieve a higher neutron detection efficiency.

\subsection{Construction Adjustments}

The relative neutron detection efficiency measurement identified two possible ${ }^{6} \mathrm{LiF}: \mathrm{ZnS}$ screen related issues, affecting the performance of SoLid.

Firstly, a particularly low $\epsilon_{\text {rel }}$ was observed in some voxels, whilst the measurements of light yield demonstrated a normal response. See figure 17. It was determined that these cubes were all wrapped using ${ }^{6} \mathrm{LiF}: \mathrm{ZnS}$ from a same batch, which was only half doped in ${ }^{6} \mathrm{Li}$. This $50 \%$ deficit in ${ }^{6} \mathrm{Li}$ was causing the lower neutron detection efficiency in these voxels, which were subsequently replaced using new lithium sheets.

The second issue involved one cube from the 12800 voxels that were tested. It was found that the cube was wrapped with only one ${ }^{6} \mathrm{LiF}: \mathrm{ZnS}$ screen instead of two, causing a low neutron detection efficiency.

The QA process provided an early identification of these two major issues and a number of minor problems, allowing the time for corrective action prior to assembly at BR2. Thanks to the stringent and all encompassing QA process, an excellent performance and homogeneity for the entire detector volume of the SoLid detector was achieved. 

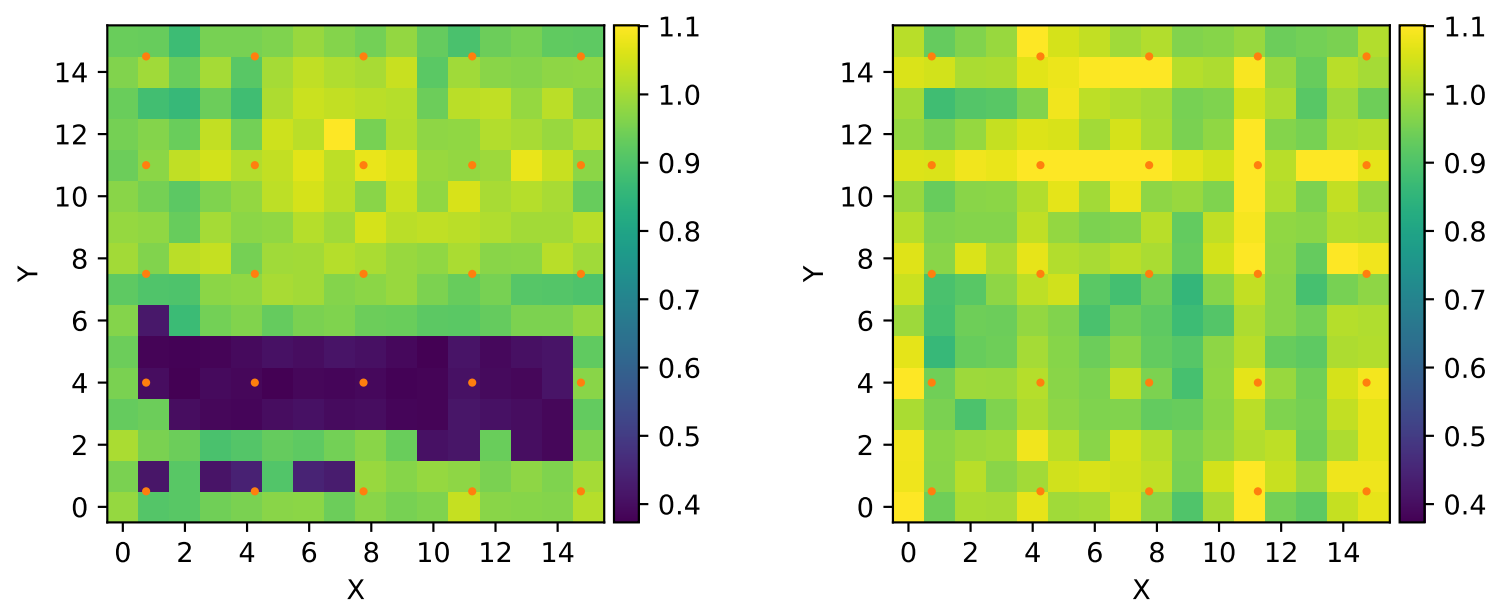

Figure 17. Orange dots are source positions. Left : Relative NS detection efficiency per cube after data merging for a plane with problematic cubes, scanned with Cf. Right : corresponding NS detection efficiency per cube after replacing cubes containing defective Li screens.

Figure 16 shows the relative NS detection efficiency among the 50 planes of the SoLid detector after replacing cubes containing half doped Li screens. A good homogeneity has been reached in all the planes.

\section{Discussion and Conclusions}

A quality assurance procedure was developed and implemented during the construction of the SoLid detector. For this purpose an automated calibration system called CALIPSO was constructed. CALIPSO allowed the early identification and fixing of defective components and, provided an initial calibration of the 12800 SoLid detector voxels. Some minor problems relating to the coupling between the fibres and either the sensors or mirrors were promptly identified and fixed. In addition, a problem with a batch of ${ }^{6} \mathrm{LiF}: \mathrm{ZnS}$ screens was identified, which had been half doped with ${ }^{6} \mathrm{Li}$. This problem was rapidly corrected by replacing the problematic screens with new ones. Thus, a very good and homogeneous response for all the 12800 SoLid voxels has been achieved. This guarantees a consistent operation, uniformity of response and, overall performance of the detector once installed at the BR2 nuclear plant.

This initial calibration for quality assurance purposes provided a first estimation of the light yield and neutron detection efficiency in all of the voxels. These parameters are expected to be larger than $60 \mathrm{PA} / \mathrm{MeV} /$ cube for light yield and $65 \%$ neutron reconstruction efficiency, exceeding the initial requirements and previous estimations reported by the SoLid collaboration [22]. Thanks to the QA process, we have shown that the construction of such a novel segmented hybrid detector, with a total target mass of $1600 \mathrm{~kg}$ has been successfully conducted.

The full SoLid detector was commissioned at the BR2 nuclear plant, at the beginning of 2018 . The detector is now operational and taking data in stable conditions. 


\section{Acknowledgments}

This work was supported by the following funding agencies: Agence Nationale de la Recherche grant ANR-16CE31001803, Institut Carnot Mines, CNRS/IN2P3 et Region Pays de Loire, France; FWO-Vlaanderen and the Vlaamse Herculesstichting, Belgium; The U.K. groups acknowledge the support of the Science \& Technology Facilities Council (STFC), United Kingdom; We are grateful for the early support given by the sub-department of Particle Physics at Oxford and High Energy Physics at Imperial College London. We thank also our colleagues, the administrative and technical staffs of the SCK $\bullet$ CEN for their invaluable support for this project. Individuals have received support from the FWO-Vlaanderen and the Belgian Federal Science Policy Office (BelSpo) under the IUAP network programme; The STFC Rutherford Fellowship program and the European Research Council under the European Union's Horizon 2020 Programme (H2020-CoG)/ERC Grant Agreement n. 682474 (A. Vacheret); Merton College Oxford.

\section{References}

[1] LSND collaboration, A. Aguilar-Arevalo et al., Evidence for neutrino oscillations from the observation of anti-neutrino(electron) appearance in a anti-neutrino(muon) beam, Phys. Rev. D64 (2001) 112007, [0104049].

[2] C. Giunti and M. Laveder, Statistical Significance of the Gallium Anomaly, Phys. Rev. C83 (2011) 065504, [1006.3244].

[3] G. Mention et al., The Reactor Antineutrino Anomaly, Phys. Rev. D83 (2011) 073006, [1101.2755].

[4] T. A. Mueller et al., Improved Predictions of Reactor Antineutrino Spectra, Phys. Rev. C83 (2011) 054615, [1101.2663].

[5] P. Huber, On the determination of anti-neutrino spectra from nuclear reactors, Phys. Rev. C84 (2011) 024617, [1106.0687].

[6] Daya Bay collaboration, F. P. An et al., Improved Measurement of the Reactor Antineutrino Flux and Spectrum at Daya Bay, Chin. Phys. C41 (2017) 013002, [1607. 05378].

[7] S. Gariazzo, C. Giunti, M. Laveder and Y. F. Li, Updated Global 3+1 Analysis of Short-BaseLine Neutrino Oscillations, JHEP 06 (2017) 135, [1703.00860].

[8] Y. Ko et al., Sterile Neutrino Search at the NEOS Experiment, Phys. Rev. Lett. 118 (2017) 121802, [1610.05134].

[9] I. Alekseev et al., DANSS: Detector of the reactor AntiNeutrino based on Solid Scintillator, JINST 11 (2016) P11011, [1606.02896].

[10] A. P. Serebrov et al., Search for sterile neutrinos in the neutrino-4 experiment, JETP Lett. 105 (2017) 347-351.

[11] STEREO collaboration, H. Almazán et al., Sterile Neutrino Constraints from the STEREO Experiment with 66 Days of Reactor-On Data, Phys. Rev. Lett. 121 (2018) 161801, [1806.02096].

[12] SoLid collaboration, Y. Abreu et al., Performance of a full scale prototype detector at the BR2 reactor for the SoLid experiment, JINST 13 (2018) P05005, [1802 . 02884].

[13] PROSPECT collaboration, J. Ashenfelter et al., The PROSPECT Reactor Antineutrino Experiment, 1808.00097. 
[14] Double Chooz collaboration, Y. Abe et al., Measurement of $\theta_{13}$ in Double Chooz using neutron captures on hydrogen with novel background rejection techniques, JHEP 01 (2016) 163, [1510.08937].

[15] RENO collaboration, J. H. Choi et al., Observation of Energy and Baseline Dependent Reactor Antineutrino Disappearance in the RENO Experiment, Phys. Rev. Lett. 116 (2016) 211801, [1511.05849].

[16] Daya Bay collaboration, F. P. An et al., Measurement of the Reactor Antineutrino Flux and Spectrum at Daya Bay, Phys. Rev. Lett. 116 (2016) 061801, [1508.04233].

[17] P. Huber, NEOS Data and the Origin of the $5 \mathrm{MeV}$ Bump in the Reactor Antineutrino Spectrum, Phys. Rev. Lett. 118 (2017) 042502, [1609.03910].

[18] Daya Bay collaboration, F. P. An et al., Evolution of the Reactor Antineutrino Flux and Spectrum at Daya Bay, Phys. Rev. Lett. 118 (2017) 251801, [1704.01082].

[19] M. Dentler et al., Updated Global Analysis of Neutrino Oscillations in the Presence of eV-Scale Sterile Neutrinos, JHEP 08 (2018) 010, [1803 . 10661].

[20] H. PHOTONICS, "MPPC (Multi-Pixel Photon Counter), https://www.hamamatsu.com/resources/pdf/ssd/s13360_series_kapd1052e.pdf."

[21] SoLid collaboration, Y. Abreu et al., A novel segmented-scintillator antineutrino detector, JINST 12 (2017) P04024, [1703.01683].

[22] SoLid collaboration, Y. Abreu et al., Optimisation of the scintillation light collection and uniformity for the SoLid experiment, JINST 13 (2018) P09005, [1806. 02461].

[23] SoLid collaboration, N. Ryder, The SoLid Anti-Neutrino Detector's Read-Out System, in Proceedings, 2016 IEEE Nuclear Science Symposium and Medical Imaging Conference: NSS/MIC 2016: Strasbourg, France, pp. 1-7, 2016.

[24] SoLid collaboration, Y. Abreu et al., Commissioning and Operation of the Readout System for the SoLid Neutrino Detector, Submited to IEEE-TNS (2018), [1812 . 05425].

[25] Geant4 collaboration, S. Agostinelli et al., Geant4: A Simulation toolkit, Nucl. Instrum. Meth. A506 (2003) 250-303.

[26] E. Siciliano et al., Energy calibration of gamma spectra in plastic scintillators using compton kinematics, Nuclear Instruments and Methods in Physics Research Section A: Accelerators, Spectrometers, Detectors and Associated Equipment 594 (2008) 232 - 243.

[27] O. Klein et al., Über die Streuung von Strahlung durch freie Elektronen nach der neuen relativistischen Quantendynamik von Dirac, Z. Phys. 52 (1929) 853. 